\title{
Extreme Loads on the Mooring Lines and Survivability Mode for the Wave Dragon Wave Energy Converter
}

\author{
S. Parmeggiani ${ }^{1, *}$, J. P. Kofoed ${ }^{2}$, E. Friis-Madsen ${ }^{3}$ \\ ${ }^{1}$ Wave Dragon Ltd., London, United Kingdom \\ ${ }^{2}$ Aalborg University, Aalborg, Denmark \\ ${ }^{3}$ Wave Dragon Aps, Copenhagen, Denmark \\ *Corresponding author. E-mail: stefano@wavedragon.net
}

\begin{abstract}
One of the main challenges Wave Energy Converters have to face on the road towards commercialization is to ensure survivability in extreme condition at a reasonable capital costs. For a floating device like the Wave Dragon, a reliable mooring system is essential. The control strategy of the Wave Dragon aims at optimizing the power production by adapting the floating level to the incoming waves and by activating the hydro-turbines and regulating their working speed. In extreme conditions though, the control strategy could be changed in order to reduce the forces in the mooring system, lowering the design requirements with almost no added cost. The paper presents the result of the tank testing of a 1:51.8 scale model of a North Sea Wave Dragon in extreme wave conditions of up to 100 years of return period. The results show that the extreme loads in the main mooring line can be reduced by approximately $20-30 \%$ by lowering the crest level and balancing the device to lean a little towards the front.
\end{abstract}

Keywords: Wave Dragon, Wave Energy Converter, Survivability, Mooring system, Control strategy

\section{Introduction}

Wave Energy Converters (WECs) have to withstand extreme events that put very high standards on their design requirements, increasing capital costs. From an economical point of view, such expenditure can be justified only by high performance in the often mild operational conditions where these devices operate for the main part of their lifetimes. One of the challenges the industry has to face in this early phase to help commercialization is therefore to jointly reduce the capital expenditures due to the survivability in extreme conditions and increase the performance in operational conditions. An efficient control strategy can help to meet both requirements with very low added cost.

\subsection{The Wave Dragon WEC - Mooring system and control strategy}

The Wave Dragon (WD) is a floating, slack-moored WEC of the overtopping type. Incoming waves are focused by two wing reflectors towards a ramp where they surge up and overtop into a reservoir placed at a higher level than the Mean Water Level (MWL). Energy is extracted as the stored water is led back to the sea through a set of low head hydro-turbines.

For an off-shore floating device like the WD the mooring system represents one of the main components ensuring the survivability. The mooring system of the WD consists of slack mooring chains of equal length distributed in circular spread, see Fig. 1. These are connected to a Catenary Anchor Leg Mooring (CALM) buoy, which again is connected to the WD platform and wings. An additional single mooring line can be connected to the rear of the platform to limit the excursions of the device.

The control strategy of the WD has three components: in a time scale of hours, the first one is aimed at optimizing the floating level of the device according to the incoming wave height in order to maximize the overtopping flow; in the time scale of minutes, the second one is the on/off regulation of the propeller turbines, which ensures a high storage efficiency of the 
reservoir; finally, in the time scale of seconds, the third one is the speed control of the turbines to ensure a constant high efficiency of the turbine-generators.

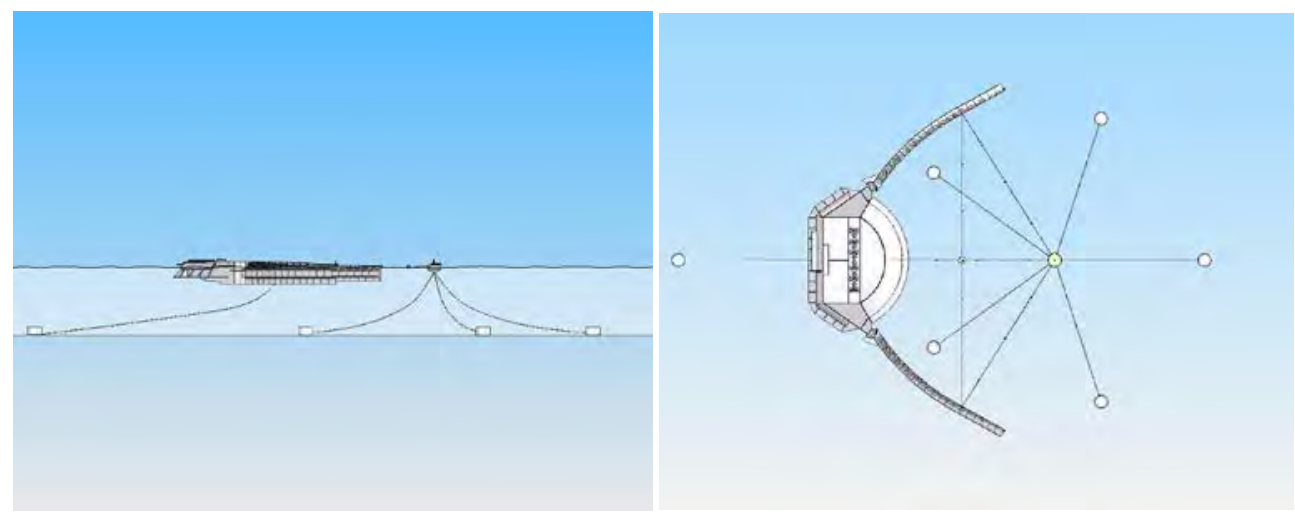

Fig. 1. Conceptual mooring system of the Wave Dragon WEC.

In extreme conditions the goal of the control strategy should no longer be to optimize the performance, but to limit the forces in the mooring system and in the structure in general. In this sense by keeping the floating level low the forces in the mooring line connecting the device to the CALM buoy, also called the main mooring line, can be decreased. This kind of control, which is hereafter referred to as the survivability mode of the $\mathrm{WD}$, can help reducing the design requirements on the mooring system.

The paper presents the results of an experimental investigation conducted on the 1:51.8 scale model of a North Sea WD to assess the efficiency of the mentioned control strategy. Different wave and setup conditions have been tested and their influences on the forces in the main mooring line and on the dynamic response of the device have been established.

In the following the tests and data analysis procedure used are presented. From the results the efficiency of the survivability mode is assessed and important considerations regarding the stability of the device are drawn. Finally, the main conclusions and future work required are presented.

\section{Method}

The study has been conducted through the wave tank testing of a scale model of the WD at the deep water basin of the Hydraulic and Coastal Laboratories of Aalborg University during October 2010.

\subsection{Test setup}

The model tested is at 1:51.8 length scale of a North Sea WD, which has a rated power of 4 MW in a wave climate of $24 \mathrm{~kW} / \mathrm{m}$. The proposed mooring system was schematically reproduced, connecting the model to an anchor at the front through the main mooring line, the stiffness of which was modeled by means of a spring to deliver the horizontal compliance. Two mooring lines at the back have been used with the only purpose of keeping the device in position.

The forces in the main mooring line $(F)$, as well as the movements of the device in surge $(S)$, heave $(H)$ and pitch $(P)$, have been recorded during the tests. 


\subsection{Tested conditions}

The wave states considered are extreme waves with return period of 10, 50 and 100 years typical of the Danish part of the North Sea. The number of wave states tested has been increased by considering for each of them three different values of peak wave steepness $S_{p}=$ $H_{s} / L_{p}(-)$, being $H_{s}(\mathrm{~m})$ the significant wave height and $L_{p}(\mathrm{~m})$ the peak wave length. All waves have been generated as irregular according to a JONSWAP spectrum with peak enhancement factor 3.3. The water depth considered corresponds to $33.7 \mathrm{~m}$ in full scale.

The influence of the height of the crest freeboard above the mean water level $\left(R_{c}\right)$ and of the directionality of the waves, expressed through the $s$ parameter of the $\operatorname{Cos}^{2 s}$ spreading function, have been investigated leading to a total of 42 tests. The values of the parameters considered in the study are resumed in Table 1, where the wave states are described by their $H_{s}$ and peak period $T_{p}$.

Table 1. Summary of the parameters considered in the study (values are given in full scale).

\begin{tabular}{|c|c|c|}
\hline Parameter name & Description & Values \\
\hline$T_{r 10}$ & $\begin{array}{l}\text { Wave with return period of } \\
10 \text { years }\end{array}$ & $H_{s}=8 \mathrm{~m}, T_{p}=13.1 \mathrm{~s}$ \\
\hline$T_{r 50}$ & $\begin{array}{l}\text { Wave with return period of } \\
50 \text { years }\end{array}$ & $H_{s}=9 \mathrm{~m}, T_{p}=13.8 \mathrm{~s}$ \\
\hline$T_{r 100}$ & $\begin{array}{l}\text { Wave with return period of } \\
100 \text { years }\end{array}$ & $H_{s}=10 \mathrm{~m}, T_{p}=14.5 \mathrm{~s}$ \\
\hline$S_{p}(-)$ & Peak wave steepness & $\begin{array}{c}S_{p 0}: \text { standard wave state } \\
S_{p+1}: H s \text { increase of } 0.5 \mathrm{~m} \\
S_{p-1}=T p \text { increase of } 1 \mathrm{~s}\end{array}$ \\
\hline$s(-)$ & Spreading coefficient & $\begin{array}{c}s_{1}=20 \text { (2D waves) } \\
s_{2}=2 \text { (3D waves) } \\
s_{3}=10 \text { (mildly 3D waves) }\end{array}$ \\
\hline$R_{c}(\mathrm{~m})$ & Crest level above MWL & $\begin{array}{l}R_{c 1}=4 \mathrm{~m} \\
R_{c 2}=3 \mathrm{~m} \\
R_{c 3}=2 \mathrm{~m} \\
R_{c 4}=1 \mathrm{~m}\end{array}$ \\
\hline
\end{tabular}

\subsection{Floating stability}

During the tests it has been observed that the model had a natural tendency to trim backwards. This behavior was found to affect the recorded forces too, as the less stable the device was, the higher were the forces. Its influence was found to be comparable to the one due to the $R_{C}$ modifications and was therefore also investigated. Following this, some modifications to the model lead to consider one setup with high stability for each $R_{c}$, as the floating level was well maintained horizontally in average, and one with low stability.

\subsection{Data analysis}

For both forces and movements the extreme values are estimated as the average of the $1 / 250^{\text {th }}$ of the highest values recorded for each time series, denoted $X_{1 / 250}, X$ being the variable considered. Other statistical values such as the mean value $X_{m}$ and standard deviation $X_{\text {stdev }}$ have been used in the data analysis. As these quantities have been evaluated on records of 30 min, corresponding in average to 1000 waves, their reliability is considered good. 
In each of the cases tested the $R_{c}$ has been derived from the mean heave by applying a vertical offset according to the model geometry. The waves have been recorded by a $2 \mathrm{D}$ rig of 7 wave gauges. The analysis of the wave records allowed to separate the incident and reflected components according to the Mansard-Funke method. The first one has been the only considered in the data analysis, characterized by the values of $H_{s}, T_{p}$ and $s$.

\section{Results}

In the following the most significant results of the tests are shown in a non-dimensional form. The extreme forces are presented as

$$
F_{n d}=\frac{F_{1 / 250}}{\rho \cdot g \cdot H_{m 0} \cdot A_{c}}(-)
$$

where $H_{m 0}(\mathrm{~m})$ is the significant wave height derived from the frequency domain analysis and $A_{c}\left(\mathrm{~m}^{2}\right)$ is the cross sectional area of the ramp of the WD, calculated as the product of average ramp width and total height (from crest to draft).

The non-dimensional heave and surge are calculated respectively as $H_{n d}=H_{1 / 250} / H_{m 0}(-)$ and $S_{n d}=S_{1 / 250} / H_{m 0}(-)$, while the pitch is directly considered as $P_{1 / 250}$ (deg).

In order to consider both the dependency on $R_{c}$ and on $L_{p}$ the independent variable chosen is the non-dimensional product of $S_{p} \cdot R=R_{c} / L_{p}(-) . R=R_{c} / H_{s}(-)$ is the non-dimensional crest level, a parameter usually considered when dealing with overtopping.

The reference system has been chosen so that displacements in surge are positive in the direction of the wave propagation, those in heave upwards and the rotations in pitch as they lower the back of the device.

The $R_{c}$ tested have been grouped in High $\left(R_{c 1}, R_{c 2}\right)$, Mid $\left(R_{c 3}\right)$ and Low $\left(R_{c 4}\right)$. As for both High and Low $R_{c}$ no significant difference was observed in the results between low and high stability, all the tests have been grouped into a total of 4 dataset for the data analysis: High $R_{c}$, Mid $R_{c}$-low stability, Mid $R_{c}$-high stability and Low $R_{c}$.

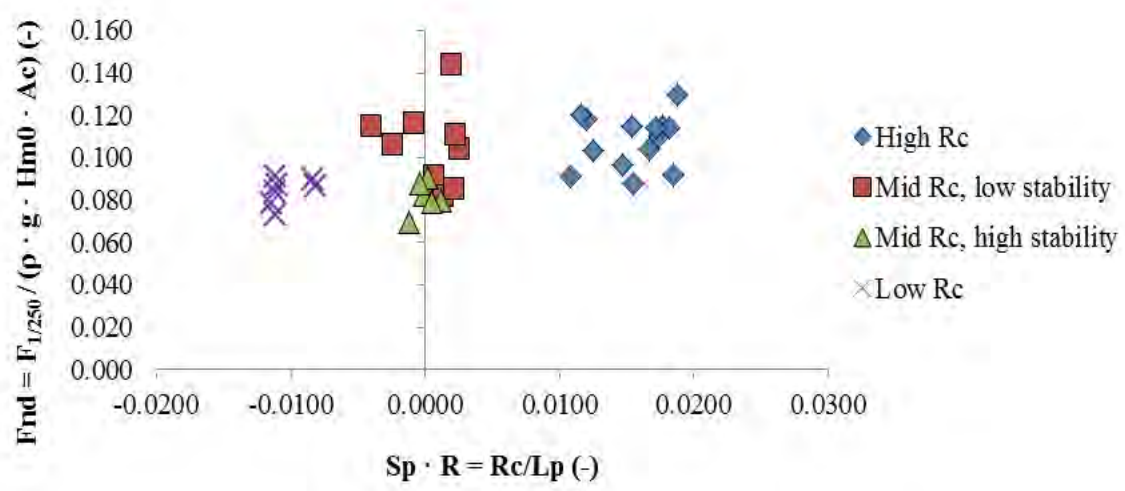

Fig. 2. Non-dimensional extreme forces in the main mooring line for $2 \mathrm{D}$ waves $\left(\mathrm{s}_{1}\right)$. 


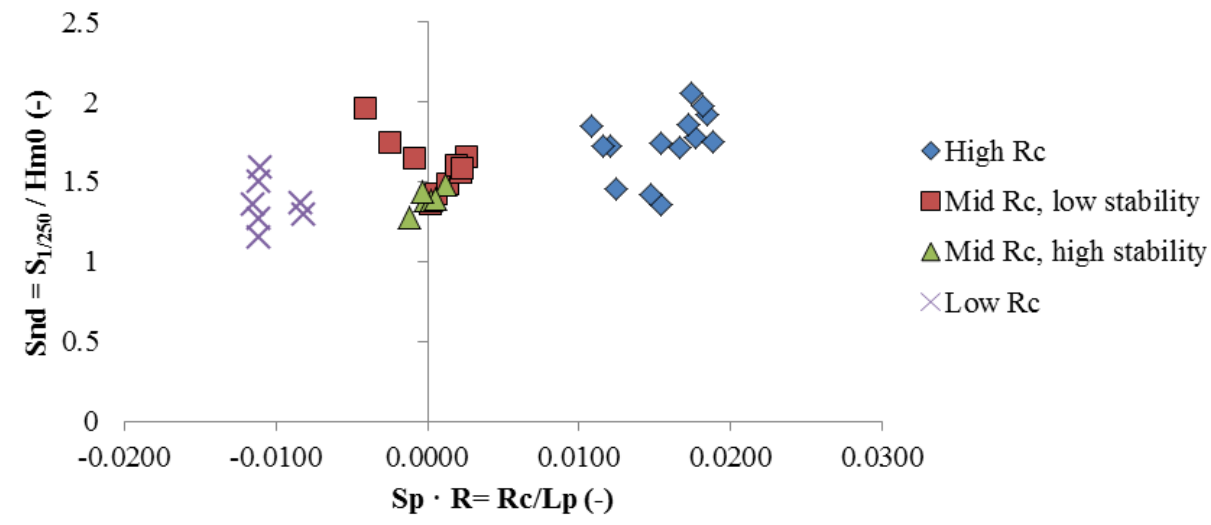

Fig. 3. Non dimensional extreme response in surge for $2 D$ waves $\left(s_{1}\right)$.

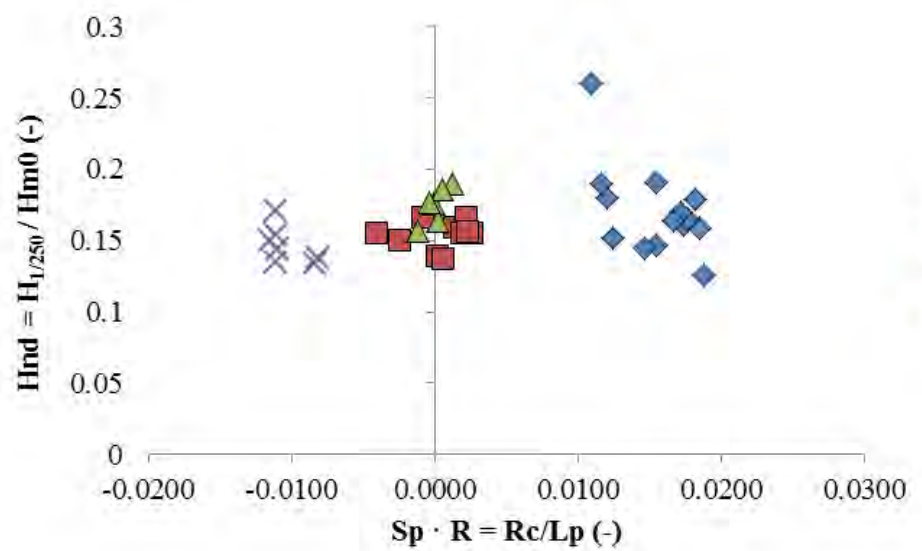

$\diamond$ High Rc

$\square$ Mid Rc, low stability $\triangle$ Mid Rc, high stability Low Rc

Fig. 4. Non dimensional extreme response in heave for $2 D$ waves $\left(s_{1}\right)$.

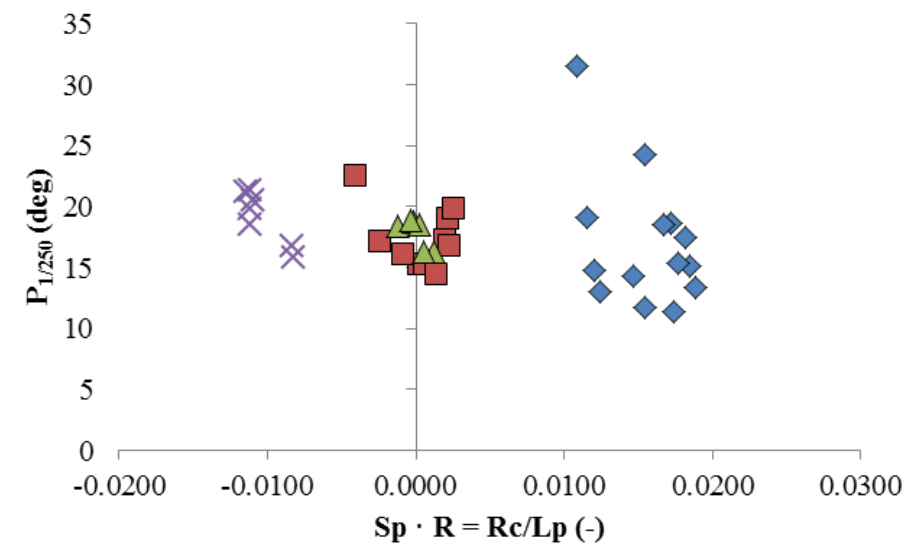

$\diamond$ high Rc

$\square$ mid Rc, low stability $\triangle$ mid Rc, high stability $\times$ low Rc

Fig. 5. Extreme response in pitch (deg) for $2 D$ waves $\left(s_{1}\right)$. 


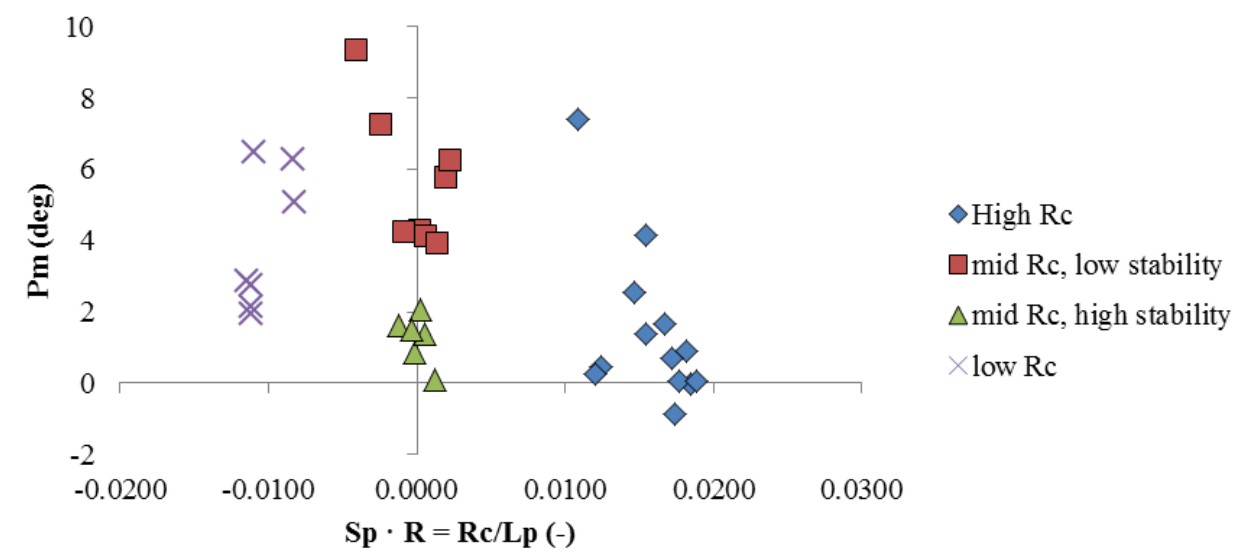

Fig. 6. Mean pitch position, or trim (deg) for $2 D$ waves $\left(s_{1}\right)$.

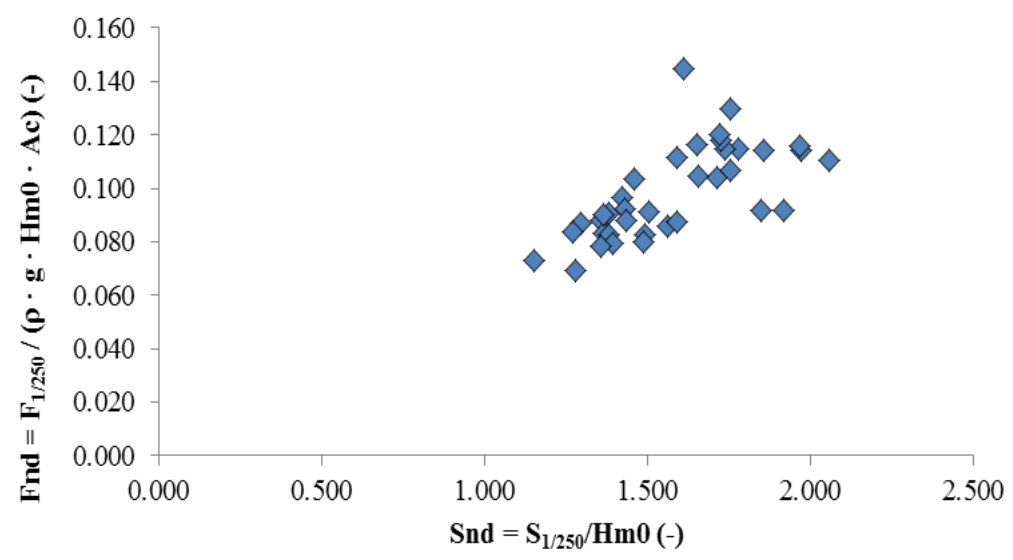

Fig. 7. Direct proportionality between non-dimensional extreme response in surge and nondimensional extreme forces in the main mooring line, for $2 D$ waves $\left(s_{1}\right)$.

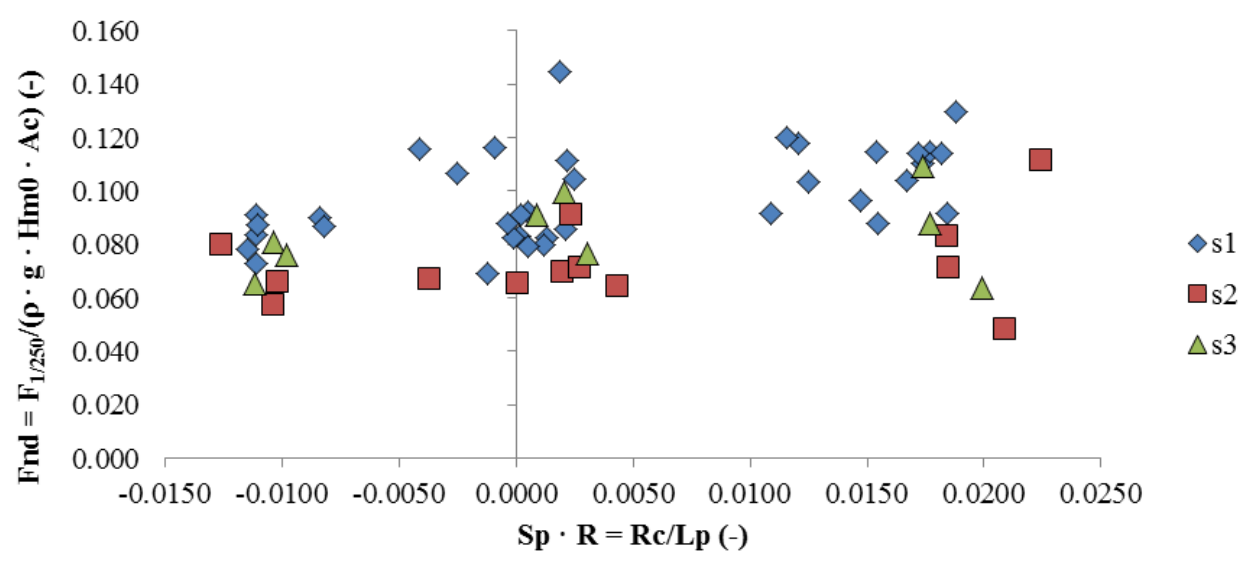

Fig. 8. Difference in the non-dimensional extreme forces in the main mooring line due to variation in the wave directionality. 


\section{Discussion}

Fig. 2 shows two very important facts. First of all the forces in the main mooring line are reduced at lower crest levels, confirming the assumption behind the proposed survivability mode. This gets to the point where it could be considered to lower the crest level even down to negative mean values, which would not make sense in terms of power production, highlighting once more how the control strategy differs switching from operational to extreme conditions.

The floating of the device at negative values of the $R_{c}$ can be explained by considering the hydrodynamics of the model during the tests: at very low floating levels the waves are completely overpassing the model, which determines a negative mean value $R_{c}$ over the test duration. Nonetheless the buoyancy of the model is still higher than its weight. Therefore as the waves stop, the $R_{c}$ is raised up again to the target (positive) floating level.

Fig. 2 also shows how the mooring forces are highly influenced by the floating stability. The forces in the Mid $R_{c}$ dataset is are fact in the order of the ones recorded at High $R_{c}$ when the stability of the device is low, while they become comparable to the ones recorded at Low $R_{C}$ as the stability is increased.

Fig. 6 shows how this behavior can be well described in terms of mean pitch, the mean position around which the device oscillates, also known as trim. Focusing on the Mid $R_{c}$ dataset, at low stability the values of $P_{m}$ are much larger than at high stability. When the mean pitch increase, the device is tilted backwards and it also increases the surface against which the waves can exert pressure on the lower part of the device; as $P_{m}$ approaches zero instead (or even as it becomes negative) proportionally more waves are hitting the ramp and as they surge it up the forces on the structure are reduced.

From a comparison of Fig. 2 and Fig. 3 it can be seen how the extreme forces in the main mooring line follow very much the extreme response in surge. This is confirmed also in Fig. 7 , where a direct proportionality between the extreme response in surge and extreme forces can be seen.

The extreme response in heave (Fig. 4) is quite constant and independent on the $R_{c}$, while the pitch shows a tendency to increase within each dataset as the $R_{c}$ lowered (Fig. 5).

Fig. 8 shows how the directionality of the waves has a significant influence on the forces. In all the cases tested the forces are reduced as the waves become 3D, due to the balancing of the components with opposite directions of the forces exerted by the waves on the device, which are not transmitted to the mooring system.

\section{Conclusions and further work}

The efficiency of the proposed survivability mode is assessed. As the floating level is lowered the extreme forces in the main mooring line can be reduced in the order of $20-30 \%$.

For the Wave Dragon this can be achieved simply by emptying the air chambers as a storm is foreseen. With no further control this condition can maintained even in the case of loss of the grid connection: a "fool-proof" passive system ensuring a high survivability.

The pitch stability of the device also plays an important role in the determination of the mooring forces, especially at intermediate $R_{c}$. In this study the stability has been described in 
average, by considering the mean value of the pitch. A reduction in this reduces the extreme forces recorded. Nevertheless it is here suggested that a more sensible parameter, possibly able to describe also the instant stability of the device, is found and used for further analysis of this behavior.

\section{Acknowledgement}

The first author acknowledges the support from the FP7 Marie Curie Actions of the European Commission, via the Initial Training Network wavetrain2 (contract-N ${ }^{\circ}$ MCITN-215414).

\section{References}

[1] J. P. Kofoed, P. Frigaard, Development of Wave Energy Devices: the Danish case, Journal of Ocean Technology, Vol. 4, No 2, 2009.

[2] NIRAS, Wave Dragon 1:4.5: up-scaling of mooring system, EU contract ENK5-CT2002-00603, Work package 2.6, deliverable 33, 36\& 43, September 2006.

[3] J. Tedd et al., Model Testing of Forces in the Reflector Joint and Mooring Forces on Wave Dragon, DCE Technical Report No. 27, Aalborg University, Department of Civil Engineering, 2005.

[4] T. Hald, J. Lynggaard, Hydraulic Model Tests on Modified Wave Dragon, Technical Report, Project No. ENS-51191/00-0067, Aalborg University, Department of Civil Engineering, 2001.

[5] J. W. Kamphuis, Introduction to coastal engineering and management, World Scientific, Advanced series on Ocean Engineering, Volume 16, 2000 